\title{
Inter Vehicle Distance based Connectivity Aware Routing in Vehicular Adhoc Networks
}

\author{
Ahmed Nazar Hassan ${ }^{1}$, Omprakash Kaiwartya ${ }^{1 *}$, Abdul Hanan Abdullah ${ }^{1}$, Dalya Khalid Sheet ${ }^{1}$, Ram \\ Shringar $\mathbf{R a o}^{2}$ \\ ${ }^{1}$ Faculty of Computing, Universiti Teknologi Malaysia (UTM), \\ 81310 Skudai Johor, Malaysia \\ ${ }^{2}$ Department of Computer Science, IGNTU, Central University, India.
}

ahmadccniit191@gmail.com, hanan@utm.my; dahmadccniit@gmail.com,rsrao08@yahoo.in

*Corresponding Author: omprakash@utm.my

\begin{abstract}
-
Connectivity in vehicular traffic environment has witnessed significant attention due to the direct impact on the performance of most of the traffic safety applications of Intelligent Transport System (ITS). Various parameters such as density, speed, direction, link quality and Inter Vehicle Distance (IVD) have been utilized for measuring connectivity. IVD has greater impact on connectivity and controls the impact of other parameters. Usage of Real time IVD for measuring connectivity has not received sufficient attention in VANETs. This paper proposes Inter Vehicle Distance based Connectivity Aware Routing (Ivd-CAR) for enhancing connectivity aware data dissemination. IVD calculation is robust and can effectively handle instantaneous GPS failure. Two localization techniques; namely, Cooperative Localization (CL) and Geometry based Localization (GL) are developed. Standard deviation of real time IVDs of a forwarding path is derived. Distribution of IVDs of a forwarding path is employed for estimating connectivity. Segment vehicle based Next Hop Vehicle (NHV) selection is utilized for incorporating network load, link quality and direction into consideration while selecting forwarding path. Simulations are carried out in ns2 to evaluate the performance of Ivd-CAR in realistic traffic environment. Comparative analysis of simulation results attests the superiority of Ivd-CAR to the state-of-the-art techniques: CSR and A-CAR.
\end{abstract}

Keywords- Connectivity aware routing; Inter vehicle distance; Connectivity; Routing; VANETs; Localization;

\section{Introduction}

Research in VANETs has received remarkable attention in recent past for addressing the issues related to on-road transportation. [1-5]. Most of the modern Intelligent Transportation Systems (ITS) applications requires an operational framework which is effectively provided by VANETs [6]. Modern ITS applications can be divided into two major categories; namely, onroad traffic safety applications and infotainment applications. Traffic safety application includes traffic monitoring through cooperative messaging, intimating blind curve road, preventing collision through automatic electronic break system, on-road real time traffic based traffic light operation and traffic light information in on-board system [7]. Infotainment application includes browsing Internet applications [8], multi-player gaming [9], music and video sharing among on-road passenger. For implementation and complete realization of these applications, efficient and reliable information dissemination in vehicular traffic environment has become one of fundamental research theme in VANETs [10]. Number of information dissemination techniques have been developed in recent past which can be broadly categorized 
into five types; namely, adhoc routing, position-based routing, cluster-based routing, broadcast routing and geocast routing [11]. Position based and geocast routing techniques have received foremost research attention for information dissemination in vehicular traffic environment due to the suitability of the techniques considering most of the traffic safety applications $[12,13]$. The significance of usage of position based and geocast routing in vehicular traffic environment was predicted by Internet Engineering Task Force (IETF) long before in 1996 [14]. In realistic on-road vehicular traffic environment, vehicles can be divided into moving platoons and traffic safety messages can be disseminated utilizing the location information of theses platoons. The same information dissemination approach is exploited in most of the aforementioned routing categories such as geocast, position and cluster based routing.

In most of the aforementioned information dissemination techniques, maintaining connectivity of forwarding path [15] and next hop vehicle selection [16] are challenging task due to the highly dynamic and self-organizing nature of vehicular traffic environment. In recent past, various attempts [17-22] have been made to address disruption in connectivity by using a number of parameters related to traffic environment for predicting durability of vehicular communication links. Some of the examples of investigation of connectivity are vehicle position information for link adaptation [17], Probabilistic link duration [18]. Road Side Unit (RSU) for connectivity [19], mobile based stations with higher transmission range for connectivity [20], path loss modelling for connectivity [21], analytical model for muti-hop connectivity [22]. Some information dissemination techniques have been also developed by exploiting the investigation on connectivity; e.g., greedy and junction based Connectivity Sensed Routing (CSR) [23], Anchor based Connectivity Aware Routing (ACAR) considering neighbour vehicles [24], Adaptive Connectivity Aware Routing (A-CAR) [25], Intersection based Connectivity Aware Routing (iCAR-II) [26]. Most of these protocols are utilizing the traffic parameters such as density, speed, transmission range and link quality except A-CAR. Statistically calculated average Inter Vehicle Distance (IVD) has been utilized in A-CAR which does not represents the real time distribution of IVD. Real time distribution of IVD is the most effective measurement of connectivity in highly dynamic vehicular traffic environment which controls the other aforementioned parameters of connectivity.

This paper proposes IVD based Connectivity Aware Routing (Ivd-CAR) protocol for enhancing connectivity aware information dissemination in VANETs. The features of IvdCAR which makes the protocol superior to the state-of-the-art protocols are provided below as contributions of the article.

- IVD calculation is robust and can effectively handle the instantaneous non-reception of location information from GPS due to modern infrastructure such as high rise buildings and fly overs or natural phenomenon such as trees along side road.

- For addressing instantaneous non-reception of location information from GPS, two localization techniques; namely Cooperative Localization (CL) and Geometry based Localization (GL) are developed. CL calculates current location of a vehicle using Received Signal Strength Indicator (RSSI) GL calculates current location using mathematical geometry.

- Standard deviation of real time IVDs information of a forwarding path is derived for predicting connectivity of the path. Standard deviation of IVDs more accurately represents the distribution of IVDs in a forwarding path.

- Segment vehicle based Next Hop Vehicle (NHV) selection is utilized for incorporating network load, link quality and direction into consideration while selecting forwarding path. 
- Performance of Ivd-CAR in realistic vehicular traffic environment is evaluated by carrying out comparative analysis of simulation results with the state-of-the-art techniques: CSR and A-CAR.

The rest of the paper is organized in the following sections. In section 2, related literature on investigating connectivity for information dissemination is qualitatively reviewed. Section 3 presents theoretical detail of the proposed Ivd-CAR protocol and its mathematical derivations. Simulation and analysis of results are discussed in Section 4. Conclusion of the article is provided in section 5 .

\section{Related Work}

In the past few years, Connectivity Aware Routing (CAR) has been in the lime light of researchers in information dissemination in VANETs. Different parameters such as speed, distance, acceleration, link quality, etc. have been used in the literature to estimate connectivity of a forwarding path while disseminating information in vehicular traffic environment. Analysis and design of an information dissemination protocol named as Connectivity-Sensed Routing (CSR) has been presented in [23]. Firstly, an analytical model for statistical estimation of inter vehicle distance has been provided. Secondly, forwarding strategy of CSR has been designed which consists of two modes; namely, segment mode based on greedy approach and intersection mode based on density aware cost function. Although statistical inter vehicle distance has been efficiently derived to model a forwarding strategy yet, non-real time inter vehicle distance, and greedy and density based forwarding modes reduces the applicability of CSR in high speed highway vehicular traffic environment.

Anchor-based Connectivity Aware Routing (ACAR) has been investigated considering neighbour vehicles in sparse and highly dynamic vehicular environment [24]. Two types of vehicular network scenario has been considered; namely, fully connected or dense and sparse vehicular network. In fully connected or dense vehicular network, Route Request Beacon Message (RRBM) and Route Reply Message (RRM) are exchanged between source and destination to establish a communication path. Source, destination or Intermediate vehicles belonging to an anchor or a junction inform their neighbouring vehicles regarding change of locations due to the directional changes taken by the vehicles. The approach of making aware the neighbouring vehicles about the changed location helps establishing reliable connectivity between source and destination. In sparse vehicular network, store and carry forward technique has been utilized considering an acceptable delay and overhead. Although the concept of awareness about change of location and sore and carry forward are promising for information dissemination in vehicular network environment yet, the absence of mathematical formulation of these concepts reduces the applicability in realistic applications.

Adaptive Connectivity Aware (A-CAR) for urban vehicular traffic environment has been suggested in [25]. Two types of connectivity model have been explored; namely, road segment and route connectivity. Road segment connectivity model is the combination of static cell based connectivity and cluster based connectivity whereas route connectivity is based on transmission quality. Routing strategy is based on prediction of location of neighbouring vehicles, selecting route adaptively and density of vehicles. Although cell and cluster based connectivity models and transmission quality based route selection are best practices in information dissemination, yet un realistic assumptions such as uniform distribution of vehicles on static cells of roads and 
density based updating of number of vehicles in a route segment reduces the chances of possible applications of the protocol in realistic vehicular traffic environment. Infrastructure and intersection based Connectivity Aware Routing (iCAR-II) has been suggested for internet access in urban VANETs [26]. A road segment between a pair of junctions is evaluated by collecting information on packet delivery delay and expected lifetime of local connectivity for including the road segment into the forwarding path from source to destination. Probability of sending control packet to collect information about a road segment and expected lifetime of local connectivity in a road segment is mathematically derived. Due to the cost of deployment of infrastructure, applicability of iCAR-II is limited to Internet access in VANETs.

Connectivity in highway vehicular traffic environment has been analytically modelled using queueing theory [27]. The analytical modelling has been carried out into two steps. In the first step, statistical distribution of inter vehicle distance has been modelled considering the number of vehicles passing through a particular point in highway scenario as Poisson process. Therefore, arrival time interval of vehicles has been considered exponentially distributed. Following these assumptions, it has been mathematically derived that inter vehicle distance follows exponential distribution in highway traffic scenario. In the second step of the modelling, connectivity of vehicular network has been analysed utilizing $M / D / \infty$ queueing system. Connectivity has been analysed in terms of expected size of platoon of vehicles and expected length of connected path between any two vehicles. Although the analytical modelling based on queueing theory and statistics is quite useful for understanding the pattern of connectivity in highway vehicular traffic environment, yet statistical estimation of inter vehicle distance does not reflect the realistic inter vehicle distance which is affected by number of parameters of vehicular traffic environment. Probability of connectivity in platoon based VANETs has been explored in [28]. It has been assumed that on-road vehicles follows Poisson distribution and inter vehicle distance follows exponential distribution. One directional and two dimensional platoon based vehicular network scenarios have been considered to derive probability of connectivity for both V2V and V2I communications. Mathematical formulation of probability of connectivity is based on number of parameters such as probability of platoon formation, transmission range of vehicles, inter vehicle distance, density of vehicles and coverage of road Side Unite (RSU). Although probability of connectivity has been effectivity derived for number of cases considering most of the parameters which affects vehicular connectivity in realistic environment, yet large number of cases makes the derivation complex in terms of implementation in a particular application.

Connectivity in fading channels of one dimensional vehicular adhoc networks has been investigated in [29]. Firstly, three fading models; namely, Rayleigh, Rician, and Weibull have been used to model the impact of fading on transmission range of vehicles. Secondly, concavity has been derived in terms of expected number of vehicles in a connected cluster of vehicles and expected length of connected communication. Derivation of connectivity has been carried out for each of the considered fading models individually. Although impact of fading on connectivity has been effectively formulated for intra-cluster connectivity yet, the absence of inter-cluster connectivity analysis reduces the applicability in realistic applications. RSUs have been utilized to insure and secure connectivity in vehicular adhoc networks [30]. Firstly, probability of connectivity has been derived in terms of transmission range, density of vehicles and number of vehicles. Critical transmission range has been obtained using the probability of connectivity derivation with threshold value consideration. Secondly, probability of number of 
reachable neighbour vehicles has been derived using Exponential distribution for time and space headways with a Robustness Factor (EwRF) to incorporate the behaviour of drivers who's vehicles does not follow these distribution. Thirdly, Generalized Extreme Value (GEV) distribution has been utilized to model inter vehicle distance in realistic vehicular traffic environment where driving behaviour is unpredictable.

Bio-inspired artificial bee colony technique has been utilized to maintain connectivity in vehicular traffic environment [31]. RSUs store the distance information of all neighbouring vehicles which is accessed by the communicating vehicles resulting in durable connectivity. Although the idea of maintaining connectivity using bio-inspired technique looks quite promising, yet non-inclusion of mathematical modelling reduces the applicability of the approach. Connectivity enhancement for spectrum-agile VANETs has been investigated considering fading channel scenario [32]. Connectivity in bi-directional traffic is derived in terms of probability of summation of channel sensing, channel switching and data exchange times is less than or equal to the time available for communication which depends on the transmission range and speed of communicating pair of vehicles. Connectivity in onedirectional traffic has been derived in terms of probability of existence of link between communicating pair of vehicles following log-normal distribution with the constraint that summation of initial separation distance and the difference between the distances travelled by communication vehicles is less than or equal to the minimum of transmission ranges of the communicating vehicles. In [33], authors have extended the connectivity estimation presented in [32] by developing algorithms for calculating communication time or time available for communication, size of message and probability of connectivity for both one and two dimensional traffic.

\section{Ivd-CAR}

In this section, inter vehicle distance based connectivity aware routing protocol has been presented for enhancing the connectivity estimation in vehicular traffic environment while disseminating traffic information. The connectivity estimation of Ivd-CAR is based on the measurement of standard deviation of inter vehicle distance of different paths from source to destination vehicle. Estimation of inter vehicle distance is a challenging task in case of erroneous GPS information at a particular instance of time. The estimation of individual inter vehicle distance addressing erroneous GPS information issue and standard deviation of inter vehicle distances of a connected path are presented in the next sections.

\subsection{Estimation of Inter Vehicle Distance}

Inter vehicle distance is one of critical parameters which has a major impact on connectivity in vehicular traffic environment. Each vehicle is assumed to be equipped with GPS receiver to get their current location. Vehicles share their location information with neighbouring vehicles. A forwarding vehicle $v_{i}$ at a location $\left(x_{i}, y_{i}\right)$ determines its distance $I V D_{i, j}$ from previous forwarder $v_{j}$ located at $\left(x_{j}, y_{j}\right)$ and updates the average inter vehicle distance $I V D_{\text {avg }}$ of the forwarding path using equation (1) and (2), respectively.

$$
\begin{aligned}
I V D_{i, j} & =\sqrt{\left\{\left(x_{i}-x_{j}\right)^{2}+\left(y_{i}-y_{j}\right)^{2}\right\}} \\
I V D_{\text {avg }} & =\frac{I V D_{\text {avg }} \times H_{\text {count }}+I V D_{i, j}}{H_{\text {count }}+1}
\end{aligned}
$$


where, $H_{\text {count }}$ represents the current hop count of the forwarding path. The aforementioned distance estimation will be useful with the prier availability of location information. In modern urban infrastructure, a situation may occur where an on-road vehicle is not able to acquire GPS location; e.g., while passing through a tunnel or on-road segment surrounded by high-rises buildings, multi floor flyovers, and multi-floor bridges. In these situations GPS based estimation of IVD may not be workable and it is either not able to estimate IVD or provides erroneous information. Two techniques are developed; namely, Cooperative Localization (CL) and Geometry based Localization (GL) for addressing this situation specific issue. These techniques are described in detail in next sections.

\subsubsection{Cooperative Localization}

In Cooperative Localization, the concept of Received Signal Strength Indicator (RSSI) based distance estimation is utilized. If RSSI is greater than a threshold value; say $\delta$, then CL is utilized for location estimation. In CL, a forwarding vehicle $v_{i}$ which is not able to receive GPS location information estimates $I V D_{i, j}$ from the previous forwarder $v_{j}$ located at $\left(x_{j}, y_{j}\right)$ using shadowing path loss model as expressed in Equation (3).

$$
I V D_{i, j}=c_{1} \times d_{0} \times e^{\left(\frac{10 \log _{10} c_{2}-\tau-\frac{P W_{r}}{P W_{t}}}{10 \times \omega}\right)}
$$

where, $c_{1}=\log _{e}(10)$ represents a constant, $d_{0}$ is the reference distance for antenna, $c_{2}$ represents a constant for antenna characteristics and channel attenuation, $\tau$ represents Gaussian random variable, $P W_{r}$ and $P W_{t}$ represents received and transmitted signal power respectively and $\omega$ is the path loss exponent.

\subsubsection{Geometry based Localization}

In case of $\mathrm{RSSI}<\delta$, inter vehicle distance estimation by CL is not accurate due to the higher interference and reflection [34]. Therefore, geometry based localization is utilized which estimates current location of a vehicle by applying mathematical geometry with past and present information about the vehicles. In GL, the information related to forwarding vehicle $v_{i}$, such as last known GPS location $\left(x_{i}^{\prime}, y_{i}^{\prime}\right)$, speed $s_{i}^{\prime}$, direction $d_{i r}^{\prime}$ are considered as past knowledge and the information such as current speed $s_{i}$, direction $d_{i r}$ are utilized as present knowledge while estimating current location $\left(x_{i}, y_{i}\right)$. The estimation is carried out considering two cases. In the first case, present $d_{i r}$ and past $d_{i r}^{\prime}$ directions are considered either same or opposite. In the second case, present and past directions are considered different (other than opposite). Estimation of current location $\left(x_{i}, y_{i}\right)$ considering both the cases are described below in detail.

In the first case, present $d_{i r}$ and past $d_{i r}^{\prime}$ directions are considered either same or opposite, the current location estimation of a forwarding vehicle is converted into a geometrical problem as shown in Figure 1. In this problem, $\left(x_{i}, y_{i}\right)$ and $\left(x_{i}^{\prime}, y_{i}^{\prime}\right)$ represent the present and past locations, $d_{i r}$ and $d_{i r}^{\prime}$ represent the present and past directions and $D$ represents the distance between present and past locations. By using the time and speed information, distance $D$ can be calculated as expressed by Equation (4).

$$
D=\left\{\begin{array}{c}
\left(t_{i}-t_{i}^{\prime}\right) \times \frac{\left(s_{i}+s_{i}^{\prime}\right)}{2}, d_{i r} \cong d_{i r}^{\prime} \\
\left|\left(\frac{\left(t_{i}-t_{i}^{\prime}\right)}{2} \times s_{i}\right)-\left(\frac{\left(t_{i}-t_{i}^{\prime}\right)}{2} \times s_{i}^{\prime}\right)\right|, d_{i r} \cong-d_{i r}^{\prime}
\end{array}\right.
$$


where $t_{i}$ and $t_{i}^{\prime}$ represents the current and past time, $s_{i}$ and $s_{i}^{\prime}$ represents present and past speed, $d_{i r}$ and $d_{i r}^{\prime}$ represents present and past directions, respectively.

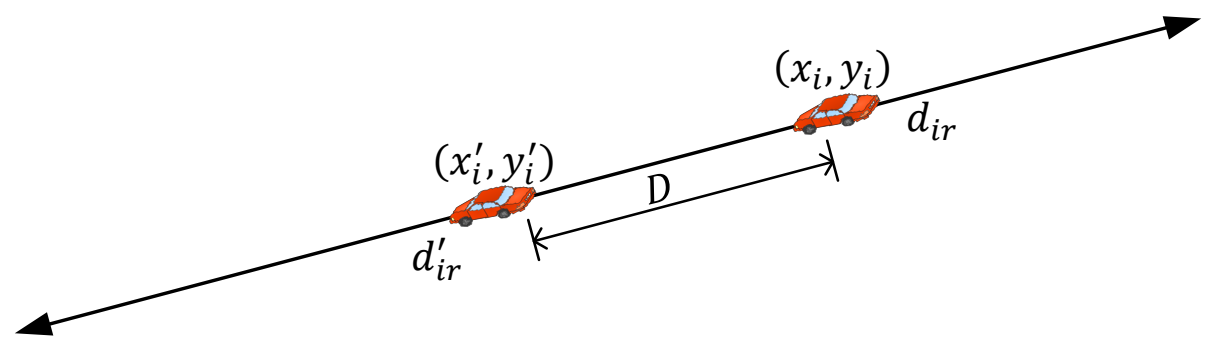

Figure 1. Finding a point in a line which is $D$ distance away from another point

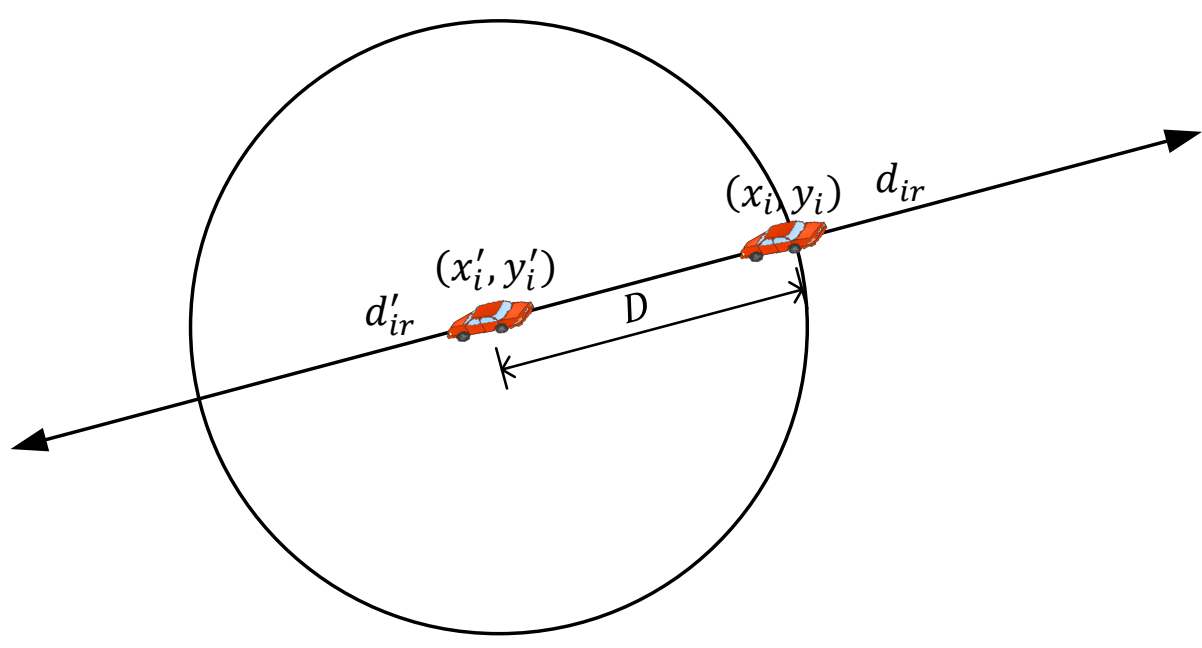

Figure 2. Finding a point on the circle with radius $D$ and center $\left(x_{i}^{\prime}, y_{i}^{\prime}\right)$

A circle of radius $D$ and center at $\left(x_{i}^{\prime}, y_{i}^{\prime}\right)$ is considered as shown in Figure 2 to solve the geometrical problem given in Figure 1. Equation of the circle can be expressed as given in Equation (5).

$$
\left(x-x_{i}^{\prime}\right)^{2}+\left(y-y_{i}^{\prime}\right)^{2}=D^{2}
$$

The current location $\left(x_{i}, y_{i}\right)$ is a point on the circle. Therefore, the point will satisfy Equation (5) as expressed in Equation (6).

$$
\left(x_{i}-x_{i}^{\prime}\right)^{2}+\left(y_{i}-y_{i}^{\prime}\right)^{2}=D^{2}
$$

Equation of a line passing through a point $\left(x_{i}^{\prime}, y_{i}^{\prime}\right)$ and having a direction (slop) $d_{i}^{\prime}$ can be expressed as given in Equation (7).

$$
\left(y-y_{i}^{\prime}\right)=d_{i}^{\prime}\left(x-x_{i}^{\prime}\right)
$$

The current location $\left(x_{i}, y_{i}\right)$ is a point on the line. Therefore, the point will satisfy Equation (7) as expressed in Equation (8).

$$
\left(y_{i}-y_{i}^{\prime}\right)=d_{i}^{\prime}\left(x_{i}-x_{i}^{\prime}\right)
$$


By simplifying Equation (6) using Equation (8) and assumption $d_{i}^{\prime}=\tan \theta^{\prime}$, current location $\left(x_{i}, y_{i}\right)$ can be calculated as expressed in Equations (9) and (10).

$$
\begin{gathered}
x_{i}=x_{i}^{\prime} \pm \mathrm{D} \cos \theta^{\prime} \\
y_{i}=y_{i}^{\prime} \pm \mathrm{D} \cos \theta^{\prime}
\end{gathered}
$$

In the second case, present and past directions are considered different (other than opposite), location estimation is converted into a geometrical problem given in Figure 3.

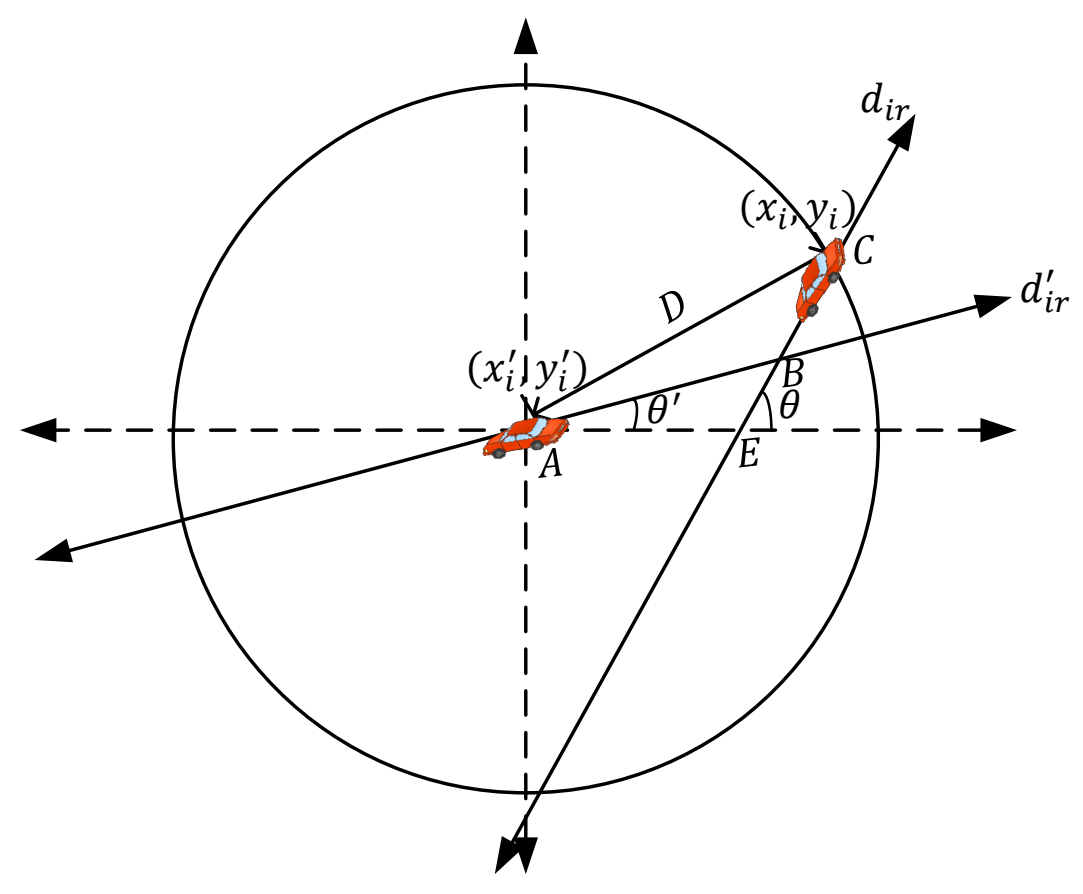

Figure 3. Finding a point on the circle intersected by a line with help of another line passing through the center of the circle and intersecting the line

In figure 3, $\angle A B C$ can be calculated as expressed in Equation (11).

$$
\begin{aligned}
\angle A B C & =180^{\circ}-\angle A B E \\
& =180^{\circ}-\left\{180^{\circ}-\left(\theta^{\prime}+(180-\theta)\right)\right\} \\
& =180^{\circ}+\theta^{\prime}-\theta
\end{aligned}
$$

In $\triangle A B C$, the distance between the vehicle $D$ can be calculated using $A B=\left(\frac{\left(t_{i}-t_{i}^{\prime}\right)}{2} \times s_{i}^{\prime}\right)$, $B C=\left(\frac{\left(t_{i}-t_{i}^{\prime}\right)}{2} \times s_{i}\right)$, and $\angle A B C=\left(180^{\circ}+\theta^{\prime}-\theta\right)$ as expressed in Equation (12).

$$
D=\sqrt{\left(A B^{2}+B C^{2}-2 \times A B \times B C \times \cos (\angle A B C)\right)}
$$

Equation of the circle can be expressed as given in Equation (13).

$$
\left(x-x_{i}^{\prime}\right)^{2}+\left(y-y_{i}^{\prime}\right)^{2}=D^{2}
$$


Equation of line $A B$ passing through a point $\left(x_{i}^{\prime}, y_{i}^{\prime}\right)$ and having a direction (slop) $d_{i}^{\prime}$ can be expressed as given in Equation (14).

$$
\left(y-y_{i}^{\prime}\right)=d_{i}^{\prime}\left(x-x_{i}^{\prime}\right)
$$

By simplifying Equation (13) using Equation (14) and considering $d_{i}^{\prime}=\tan \theta^{\prime},(x, y)$ can be calculated as expressed in Equations (15) and (16).

$$
\begin{aligned}
& x=x_{i}^{\prime} \pm \mathrm{D} \cos \theta^{\prime} \\
& y=y_{i}^{\prime} \pm \mathrm{D} \cos \theta^{\prime}
\end{aligned}
$$

The current location $\left(x_{i}, y_{i}\right)$ is a point on the circle. Therefore, the point will satisfy Equation (13) as expressed in Equation (17).

$$
\left(x_{i}-x_{i}^{\prime}\right)^{2}+\left(y_{i}-y_{i}^{\prime}\right)^{2}=D^{2}
$$

Equation of line $B C$ passing through a point $\left(x_{i}, y_{i}\right)$ and having a direction (slop) $d_{i}$ can be expressed as given in Equation (18).

$$
\left(y-y_{i}\right)=d_{i}\left(x-x_{i}\right)
$$

By using Equations (15) and (16) and considering $d_{i}=\tan \theta$, Equation (18) can be expressed as given in Equation (19).

$$
\begin{gathered}
\left(y_{i}^{\prime} \pm \mathrm{D} \cos \theta^{\prime}-y_{i}\right)=\tan \theta\left(x_{i}^{\prime} \pm \mathrm{D} \cos \theta^{\prime}-x_{i}\right) \\
\left(y_{i}^{\prime}-y_{i}\right)=\tan \theta\left(x_{i}^{\prime}-x_{i}\right) \pm \alpha
\end{gathered}
$$

Where constant $\alpha=\left(\tan \theta \mathrm{D} \cos \theta^{\prime} \pm \mathrm{D} \cos \theta^{\prime}\right)$ is considered for simplification. Equation (17) can be simplified using Equation (19) to obtain a quadratic equation in $x_{i}$ as expressed in Equation (20).

$$
\sec ^{2} \theta x_{i}^{2}+x_{i}\left(-2 x_{i}^{\prime} \sec ^{2} \theta \pm 2 \alpha \tan \theta\right)+\left(x_{i}^{\prime 2} \sec ^{2} \theta \pm \alpha^{2} \mp 2 \alpha \tan \theta x_{i}^{\prime}-D^{2}\right)=0
$$

Two roots can be obtained by solving Equations (20) as expressed in Equation (21).

$$
x_{i}=\frac{-\left(-2 x_{i}^{\prime} \sec ^{2} \theta \pm 2 \alpha \tan \theta\right) \pm \sqrt{\left(-2 x_{i}^{\prime} \sec ^{2} \theta \pm 2 \alpha \tan \theta\right)^{2}-4 \sec ^{2} \theta\left(x_{i}^{\prime 2} \sec ^{2} \theta \pm \alpha^{2} \mp 2 \alpha \tan \theta x_{i}^{\prime}-D^{2}\right)}}{2 \sec ^{2} \theta}
$$

Equation (17) can be simplified to obtain a quadratic equation in $y_{i}$ as expressed in Equation (22).

$$
y_{i}^{2}+\left(-2 y_{i}^{\prime}\right) y_{i}+\left(x_{i}-x_{i}^{\prime}\right)^{2}+\left(y_{i}^{\prime}\right)^{2}-D^{2}=0
$$

Two roots can be obtained by solving Equations (22) as expressed in Equation (23).

$$
y_{i}=y_{i}^{\prime} \pm \sqrt{\left(x_{i}-x_{i}^{\prime}\right)^{2}-D^{2}}
$$

After calculation of current location using either CL or GL, inter vehicle distance is estimated as given by Equations (1) and (2). Standard deviation calculation using the inter vehicle distance of a particular path is described in the next section.

\subsection{Standard Deviation of IVD based Forwarding}

Standard deviation of inter vehicle distance of a forwarding path is significantly important to maintain connectivity considering platoon based communication architecture in vehicular traffic environment [35]. In Ivd-CAR, sender vehicle calculates segment area $A_{s}$ for identifying 
forwarding region [36]. Forwarding region of a sender which is described in Figure 4 is calculated as given by Equation (24).

$$
A_{s}=\left(R^{2} \times \tan ^{-1}\left(\frac{L}{2 M}\right)\right)-\left(\frac{L}{4} \times \sqrt{4 R^{2}-L^{2}}\right)
$$

where $L=P Q, M=S T, \alpha=2 \times \tan ^{-1}\left(\frac{L}{2 M}\right)$ and $R$ represents the transmission range of sender vehicle.

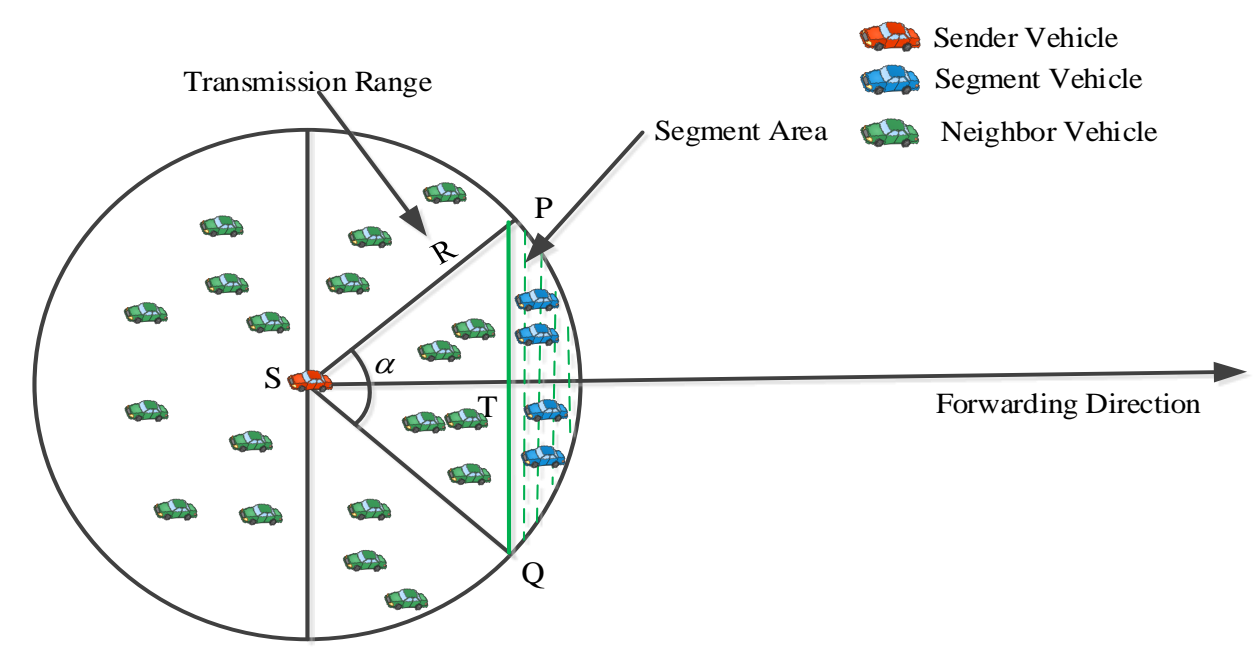

Figure 4. Segment area of a sender vehicle

Sender vehicle sends Route Request (RREQ) to the vehicles belonging to a segment area. These candidate forwarders are referred as segment vehicles. Each forwarding vehicle $v_{i}$ of RREQ estimates $I V D_{i, j}$ with previous forwarder $v_{j}$ and updates $I V D_{\text {avg }}$ of the forwarding path using the approach mentioned in Section 3.1 and inserts it into RREQ. Once the source vehicle receives a Route Reply (RREP) control message from any of the destination vehicle of the geocast region, it calculates standard deviation $S D_{I V D}$ of inter vehicle distance as expressed by equation (25).

$$
S D_{I V D}=\sqrt{\frac{1}{H_{c}} \sum_{i=1, j=2}^{i=\left(H_{c}-1\right), j=H_{c}}\left(I V D_{i, j}-I V D_{a v g}\right)^{2}}
$$

After calculating $S D_{I V D}$ of all the paths possible from each segment vehicles, forwarding next hop vehicle is selected considering the path with minimum $S D_{I V D}$. The complete geocast routing algorithm based on inter vehicle distance is developed in the next section.

\subsection{Ivd-CAGR algorithm}

In this section, the aforementioned estimation of inter vehicle distance and standard deviation of inter vehicle distances of forwarding path are utilized to develop an algorithm for connectivity aware geocast routing. Ivd-CAGR selects next hop vehicle considering the forwarding path having minimum standard deviation of inter vehicle distances. Ivd-CAGR is presented in Algorithm 1.

\section{Algorithm 1: Ivd-CAGR}

\section{Notations}

$v_{c f}$ : Current Forwarder Vehicle; $S_{s v}$ : Set of Segment Vehicles; $\alpha$ : Sector angle

$v_{d}$ : A vehicle in destination geocast region; $v_{s}$ : Original Source Vehicle

$v_{N H V}$ : Next hop Vehicle; $S_{o h}$ : Set of One hop Neighbor; $v_{i}: i^{\text {th }}$ Individual vehicle

$R R E Q_{i}$ : Route request control message for $i^{\text {th }}$ vehicle; $S_{s d}$ : Set of standard deviations 
$R R E P_{i}$ : Route reply control message from $i^{\text {th }}$ vehicle; $P_{r}$ : Received signal power; $I V D_{i, j}$ : Inter vehicle distance between $i^{\text {th }}$ and $j^{\text {th }}$ vehice; $l_{g p s}$ : Location information by GPS $I V D_{\text {avg }}:$ Average inter vehicle distance of a path

Input $R, \alpha, M, L \lambda, \delta$

$\underline{\text { Process }}$

1. initialization

$$
v_{c f}=v_{s} ; S_{o h}=\phi ; S_{s v}=\phi ; S_{s d}=\emptyset v_{N H V}=n u l l ; \alpha=90^{0}
$$

2. $S_{o h}=\left\{\right.$ neighboring vehicles of $\left.v_{c f}\right\}$

3. if $\left(v_{d} \in S_{o h}\right)$ then

Send the packet to $v_{d}$ using available direct link

$v_{d}$ broadcasts the packet within geocast region

$$
\text { exit }
$$

4. else

5. while $\left(v_{c f} \neq v_{d}\right)$

Calculate $A_{s}$ using Equation (24)

$S_{s v}=\left\{\right.$ the neighboring vehicles in $\left.A_{s}\right\}$

6. for each $v_{i} \in S_{S v}$

Transmit $R R E Q_{i}$ for searching forwarding path

7. If $\left(l_{\text {gps }}\right.$ is available)

Calculate $I V D_{i, j}$ using Equation (1)

Update $I V D_{a v g}$ of current path using Equation (2)

8. elseif $\left(P_{r}>\delta\right)$

Estimate $I V D_{i, j}$ using CL given in Equation (3)

Update $I V D_{a v g}$ of current path using Equation (2)

9. else

Estimate $I V D_{i, j}$ using GL and Equation (1)

Update $I V D_{a v g}$ of current path using Equation (2)

$$
\text { endif }
$$

\section{endfor}

10. while $\left(R R E P_{i}\right.$ is not available $\left.\forall v_{i} \in S_{s v}\right)$

$$
\text { endwhile }
$$

Wait for random amount of time for route reply

11. for each $v_{i} \in S_{s v}$

Calculate $S D_{I V D}$ using Equation (25)

\section{endfor}

$S_{s d}=S_{s d} \cup S D_{I V D}$

12 Select $v_{N H V}=$ a vehicle with minimum SD path from $\left\{S_{S d}\right\}$

13 Transmit the packet to $v_{N H V}$ and $v_{c f}=v_{N H V}$

\section{endif}

\section{endwhile}

14. exit

Output: $v_{N H V}$

\section{Simulation Results}

In this section, description of simulations carried out to evaluate the performance of Ivd-CAGR is provided. Two types of road network configuration is used for carrying out simulations. Firstly, software made road network with sixteen junction points is used in the simulations. Secondly, realistic city map based road network is used in simulations to verify the results of 
software made road configuration and to assess the performance of Ivd-CAGR in realistic road network. The metrics considered for performance evaluation are packet delivery ratio, end-toend delay, path disconnection and hop count which are very closely related with the connectivity of the network. State-of-the-art techniques: A-CAR and M-CAR are used for comparative analysis of simulation results.

\subsection{Simulation Environment}

Simulations are carried out using the open source network simulator ns-2.34 with the help a network configuration generating software MOVE. Network configuration such as number of junction points, number of lanes in each road segment, traffic lights at each junctions is created using road map editor module of MOVE. Probability of left and right turn in junction points and probability of lane changing, speed at lanes are configured using vehicle movement editor module of MOVE. Mobility trace file is created using the aforementioned configuration and utilized in ns-2 as scenario file.

Table 1. Simulation Parameters

\begin{tabular}{llll}
\hline \hline Parameters & Values & Parameters & Values \\
\hline \hline Speed interval & $60-300 \mathrm{~s}$ & Ifqlen & 50 \\
Simulation area & $3000 \times 3000 \mathrm{~m}^{2}$ & Channel type & Wireless \\
Vehicle speed & $2.8-22.4 \mathrm{~m} / \mathrm{s}(10-80 \mathrm{Km} / \mathrm{h})$ & Antenna model & Omni directional \\
Number of vehicle & $100-500$ & Propagation model & Shadowing \\
Packet senders & 15 & MAC data rate & $5 \mathrm{Mbps}$ \\
Transmission range & $300 \mathrm{~m}$ & MAC protocol & IEEE 802.11p \\
Traffic type & CBR & Hello timeout & $0.5 \mathrm{~s}$ \\
Packet type & UDP & Query period & $2.5 \mathrm{~s}$ \\
Packet size & 512 bytes & Frequency & $5.9 \mathrm{GHz}$ \\
CBR rate & 6 Packets $/ \mathrm{s}$ & Routing protocols & Ivd-CAGR \\
\hline \hline
\end{tabular}

A road network of sixteen junction points with two lanes in each road is configured. Any junction points are $1000 \mathrm{~m}$ apart and lane width is $5 \mathrm{~m}$ in simulation area. The number of onroad vehicles considered on all the lanes of the simulation area is in the range 100-500. Speed range for on-road vehicles is considered in the range $10-80 \mathrm{Km} / \mathrm{h}$. Speed change interval is one of major parameters that affect IVD. Speed change interval is considered in the rang $60-$ $300 \mathrm{~s}$. Transmission range of $250 \mathrm{~m}$, Packet size of 512 bytes, wireless channel type, traffic type as CBR, shadowing propagation model, Omni directional antenna model, 802.11p MAC protocol are the other basic parameters considered while simulating Ivd-CAGR. A summary of simulation parameter values is provided in Table- 1 and are approximately equal as considered in [36]. Simulations are performed after configuring the network and on-road traffic environment with the aforementioned values of parameters. Different source vehicle and geocast region are randomly selected from two pre-determined junction points which is kept same for all the ten simulation runs for recoding simulation points used in results. This way, average of ten different simulation runs is taken for each particular value used in results.

\subsection{Analysis of Results}

In this section, analysis of simulation results are discussed. The metrics for performance evaluation; namely, packet delivery ratio, path disconnection and hop count are measured in two major simulation scenarios. In the first scenario, metrics are measured with increasing speed of vehicles which is in the range $10-80 \mathrm{Km} / \mathrm{h}$ to analyze the impact of speed on the performance of Ivd-CAGR. In the second scenario, metrics are measured with increasing 
number of vehicles in the network which is in the range $25-200$ to analyze the impact of density of vehicles on the performance of Ivd-CAGR.

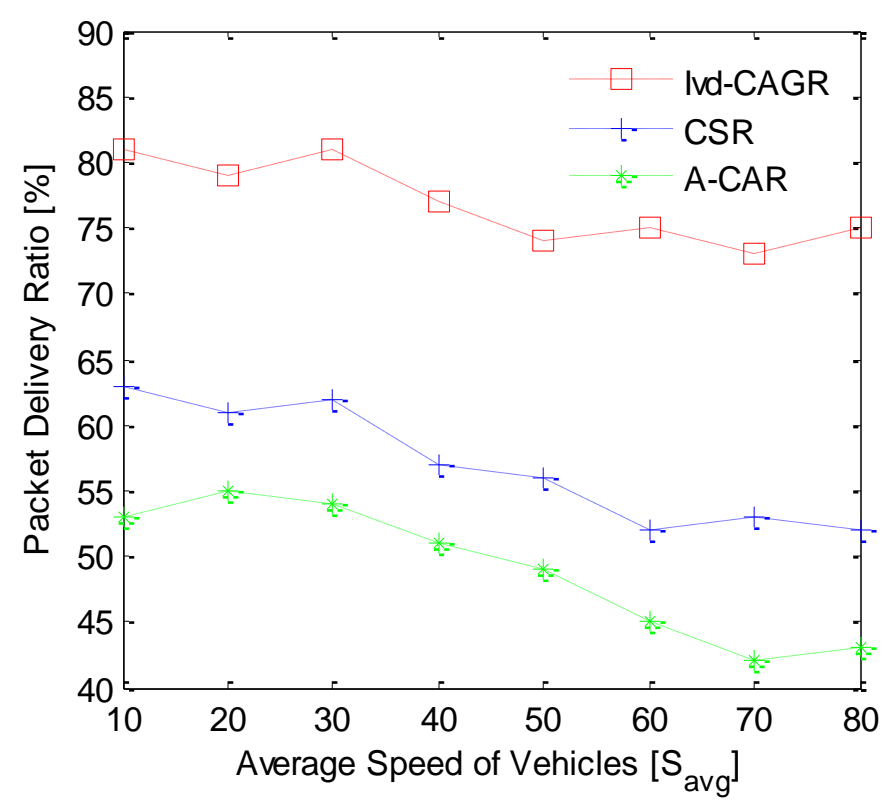

Figure 5. Impact of speed of vehicles on packet delivery ratio

Results in Figure 5 show the comparison of packet delivery ratio between Ivd-CAGR and the state-of-the-art techniques: CSR and A-CAR. It can be observed that packet delivery ratio of Ivd-CAGR is comparably higher as compared to that of the state-of-the-art techniques. In particular, packet delivery ratio of Ivd-CAGR is approximately in the range $75-82 \%$ for the considered range of speed whereas it is approximately in the range $52.6-63.7 \%$ and $55-$ $43.7 \%$ in case of CSR and A-CAR, respectively. Moreover, the rate of decrement of packet delivery ratio of Ivd-CAGR with increasing vehicle speed is lower as compared to that of stateof-the-art techniques. This can be attributed to the fact that Ivd-CAGR establishes more durable and stable path between source vehicles and destination geocast region due to the incorporation of inter vehicle distance into forwarding criteria which indirectly reduces the impact of speed of vehicles on durability and stability of a forwarding path. Estimation of path durability and stability of CSR is based on statistical estimation of inter vehicle distance which does not corresponds to the on-road real time inter vehicle distance. Estimation of path durability and stability of A-CAR is based on density of vehicles which does not guaranty the availability of path due to uneven distribution of vehicles in realistic on-road traffic scenario. Packet delivery ratio of state-of-the-art techniques degrade at higher rate as the speed of vehicles reaches above $50 \mathrm{Km} / \mathrm{h}$ due to the same reasons. 


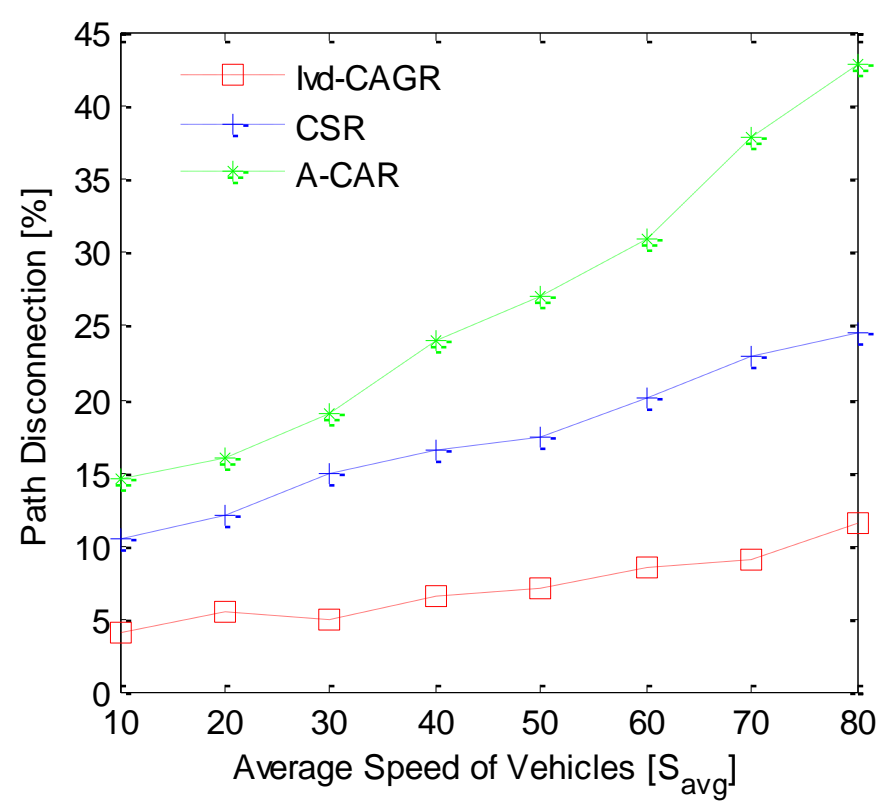

Figure 6. Impact of speed of vehicles on path disconnection

Figure 6 summarizes results of a comparative study of impact of speed of vehicles on path disconnection in Ivd-CAGR and the state-of-the-art techniques. It is clear that path disconnection of Ivd-CAGR is comparably lower in comparison with that of the state-of-theart techniques. Specifically, path disconnection of Ivd-CAGR is approximately in the range 5 $11.5 \%$, whereas it is approximately in the ranges $10.5-24 \%$ and $14-43.5 \%$ in case of CSR and A-CAR, respectively. This is due to the consideration of standard deviation of inter vehicle distance and direction for establishing a forwarding path between source and destination geocast region. The consideration makes the forwarding path robust due to the dependability of lifetime of a forwarding path on standard deviation of inter vehicle distance and direction in vehicular traffic environment. Standard deviation of inter vehicle distance are not considered for measuring connectivity of a forwarding path in both the forwarding modes; namely, greedy based segment mode and density based intersection mode. Standard deviation of inter vehicle distance as well as direction are also not considered in the connectivity estimation of A-CAR where cluster based connectivity and cell based connectivity are integrated for measuring connectivity from source to destination.

Figure 7 shows the comparison between Ivd-CAGR and the state-of-the-art techniques in terms of impact of speed of vehicles on hop count. It is clear that hop count of Ivd-CAGR is comparably lower and stable in comparison with that of the state-of-the-art techniques. Specifically, hop count of Ivd-CAGR is approximately in the range $18-24$ whereas it is approximately in the ranges $20-80$ and $53-192$ in case of CSR and A-CAR, respectively. This is due to the segment vehicle based directional forwarding of Ivd-CAGR which avoids selection of closer neighboring vehicles as next hop vehicle. Segment vehicles are not the vehicles on the border of transmission range. Segment vehicles are well inside the transmission range of forwarding vehicle, therefore, avoids disconnections while significantly reducing hop count. CSR selects next hop vehicle from the border vehicles to select farthest vehicle in segment mode, whereas density based lowest cost vehicle as next hop vehicle in intersection mode. High outage probability of border vehicles and density based cost function results in higher hop count. The consideration of static cells on road and updating number of vehicles in a road segment based on density increases hop count in case of A-CAR. 


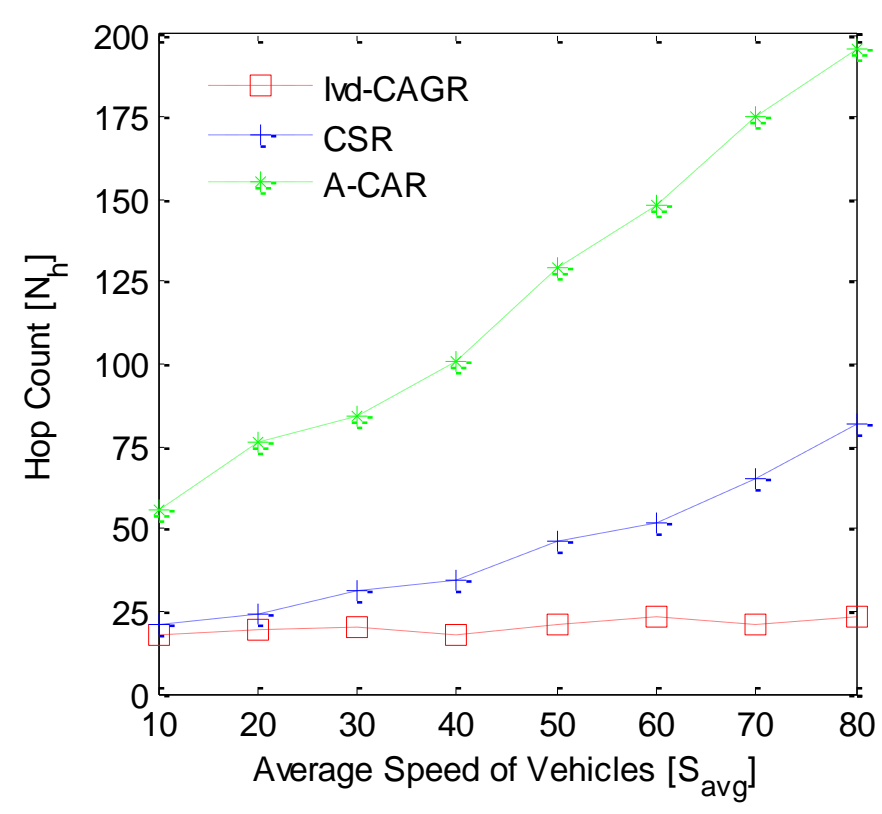

Figure 7. Impact of speed of vehicles on hop count

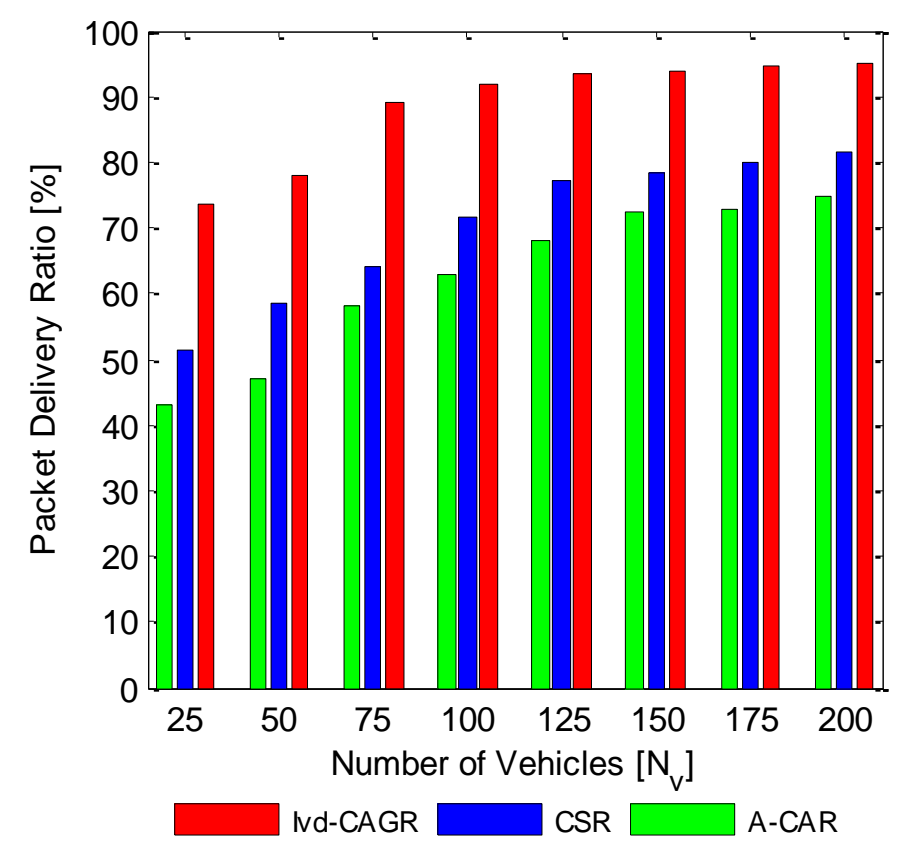

Figure 8. Impact of number of vehicles on packet delivery ratio

Figure 8 shows the comparison of impact of number of vehicles on packet delivery ratio between Ivd-CAGR and the state-of-the-art techniques. Results clearly state that packet delivery ratio of Ivd-CAGR is higher and becomes stable once the number of vehicle reaches above 75 as compared to that of the state-of-the-art techniques. In particular, packet delivery ratio of Ivd-CAGR is in the range $75-95 \%$ and becomes stable after reaching above $90 \%$, whereas it is approximately in the ranges $50-81 \%$ and $42-75 \%$ in case of CSR and ACAR, respectively. This is because of the stability of the forwarding path identified by Ivd- 
CAGR, due to the consideration of inter vehicle distance. The forwarding path becomes stable quickly once the sufficient number of vehicles becomes available in the network; i.e., 75 in the considered network scenario. Statistically estimated inter vehicle distance based forwarding path selection and density based number of vehicle estimation forwarding path selection are responsible for the lower packet delivery ratio in case of CSR and A-CAR, respectively. Statistical estimation does not reflect the real time inter vehicle distance and density based number of vehicle estimation does not reflect the exact number of vehicles.

Figure 9 presents the comparison of impact of number of vehicles on path disconnection between Ivd-CAGR and the state-of-the-art techniques. Results clearly state that path disconnection of Ivd-CAGR decreases with the increase in number of vehicles whereas it rapidly increases in case of CSR and A-CAR. Specifically, path disconnection of Ivd-CAGR decreases with the increaser in number of vehicles in the range 25-150 vehicles and stabilizes approximately at $5 \%$ once the number of vehicles reaches above 150 vehicles. This is due to the decrement in standard deviation of inter vehicle distance with in number of vehicles in the network. Smaller standard deviation of inter vehicle distance results into more robust forwarding path. Standard deviation of inter vehicle distance is not considered in CSR and ACAR, therefore, probability of path disconnection increases with the increases in number of vehicles. Additionally, direction is also not considered in forwarding path selection which results into higher path disconnection in case of A-CAR.

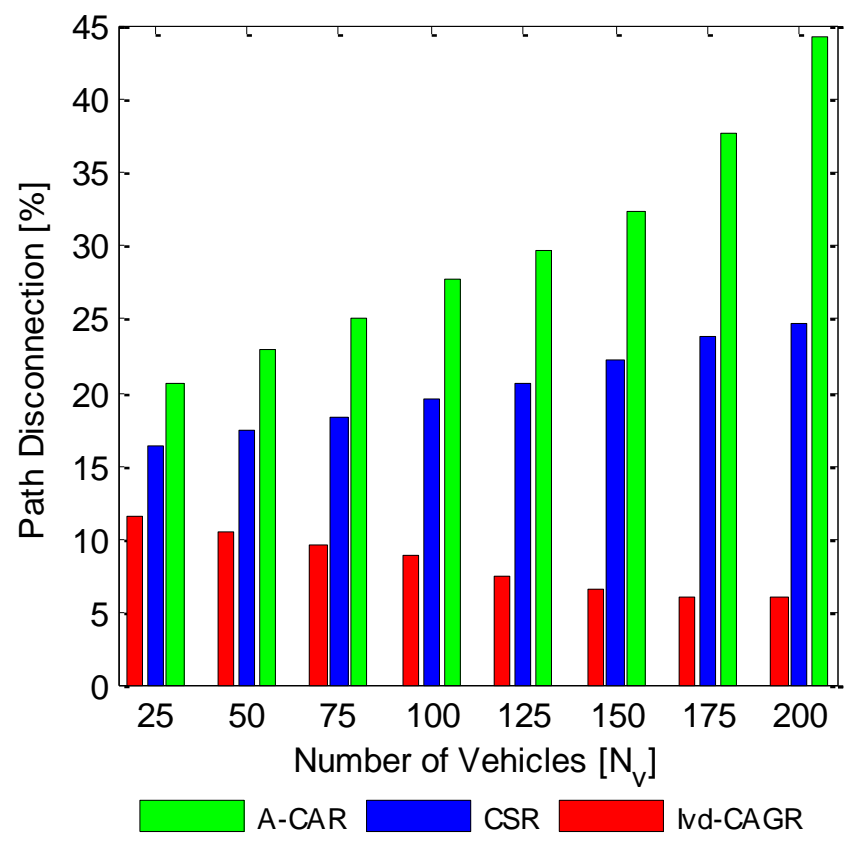

Figure 9. Impact of number of vehicles on path disconnection 


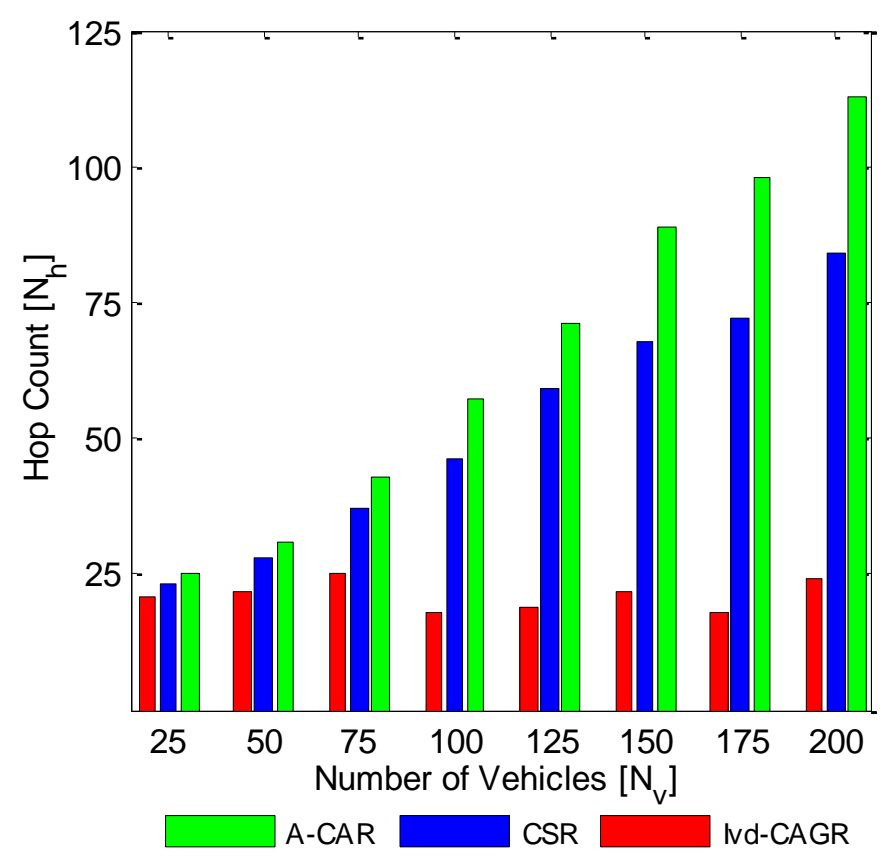

Figure 10. Impact of number of vehicles on hop count

Figure 10 shows the comparison between impact of number of vehicles on hop count in IvdCAGR and the state-of-the-art techniques. It can be clearly observed that hop count of IvdCAGR is significantly smaller as compared to that of the state-of-the-art techniques. In particular, hop count of Ivd-CAGR varies approximately in the range $18-25$, whereas it rapidly increases in the ranges $24-85$ and $25-115$ with the increase in number of vehicles in case of CSR and A-CAR, respectively. This can be attributed to the fact that Ivd-CAGR uses segment vehicle based directional forwarding which selects appropriate single next hop vehicle from segment vehicles without considering the number of neighboring vehicles. Segment vehicle based forwarding path selection technique significantly reduces hop count without being affected from number of vehicles in the network. Greedy based farthest vehicle selection in segment mode and density based lowest cost vehicle selection in intersection mode causes bigger hop count in case of CSR whereas integration of road segment selection with multi hop path selection in each road segment without considering the length of the path is responsible bigger hop count in case of A-CAR.

\section{Conclusion}

In this paper, Inter Vehicle Distance based Connectivity Aware Routing (Ivd-CAR) has been presented. Cooperative Localization (CL) and Geometry based Localization (GL) have been developed. Standard deviation of real time IVDs of a forwarding path has been derived for estimating connectivity. Segment vehicle based Next Hop Vehicle (NHV) selection has been utilized. It has been observed that CL and GL make the calculation of IVD robust from instantaneous GPS failure. Standard deviation of IVDs more accurately represents the distribution of IVDs in a forwarding path which enables Ivd-CAR to select stable and durable forwarding path resulting in higher packet delivery ratio. Segment vehicle based NHV selection reduces hop count without affecting the quality of connectivity resulting in lower end-to-end delay. Robust IVD calculation reduces the probability of path failure resulting in lower rate of path disconnection. Evolutionary technique based NHV selection in Ivd-CAR to incorporate 
the impact of more number of traffic parameters on forwarding path selection will be explored in future work.

\section{Acknowledgements}

The research is supported by Ministry of Education Malaysia (MOE) and conducted in collaboration with Research Management Center (RMC) at University Teknologi Malaysia (UTM) under VOT NUMBER: Q.J130000.2528.06H00.

\section{References}

[1] Stanford University. "New wireless technology developed for faster, more efficient networks," ScienceDaily, 15 February 2011

www.sciencedaily.com/releases/2011/02/110214155503.htm

[2] US Department of Transportation "Research at National Highway Traffic Safety Administration (NHTSA)," USA. http://www.nhtsa.gov/Research/

[3] Japan New Car Assessment Program (JNCAP) and Advanced Safety Vehicle Program(ASV) "National Agency for Automotive Safety and Victims' Aid," Japan. http://www.nasva.go.jp/index.html

[4] European Commission "Transport-Research and Innovation", Europe. http://ec.europa.eu/research/transport/projects/index_en.htm

[5] "IEEE Standard for Information technology-Telecommunications and information exchange between systems-Local and metropolitan area networks-Specific requirements Part 11: Wireless LAN Medium Access Control (MAC) and Physical Layer (PHY) Specifications Amendment 6: Wireless Access in Vehicular Environments," IEEE, pp. 1-51, 2010.

[6] W. Liang, Z. Li, H. Zhang, S. Wang, and R. Bie, "Vehicular ad hoc networks: architectures, research issues, methodologies, challenges, and trends," International Journal of Distributed Sensor Networks, 14(1), pp. 112, 2014.

[7] L. Li, D. Wen, and D. Yao, “A survey of traffic control with vehicular communications, ” IEEE Transaction on Intelligent Transportation Systems, 15(1), pp. 425-432, 2014.

[8] A. Benslimane, S. Barghib, and C. Assi, “An Efficient Routing Protocol for Connecting Vehicular Networks to the Internet", Pervasive and Mobile Computing, 7(1), pp. 98-113, 2011.

[9] O. K. Tonguz, and M. Boban, "Multiplayer Games over Vehicular Ad-Hoc Networks: A New Application", Ad Hoc Networks, 8(1), pp. 531-543, 2010.

[10]M. Gerla, C. Wu, G. Pau, and X. Zhu, "Content distribution in VANETs," Vehicular Communications, 1(1), pp. 3-12, 2014

[11]C. Suthaputchakun, and Z. Sun, “Routing protocol in inter vehicle communication systems: a survey,” IEEE Communications Magazine, 49(12), pp.150-156, 2011.

[12]O. Kaiwartya and S. Kumar, "Geocast routing: Recent advances and future challenges in vehicular adhoc networks," In Proceedings of the International Conference on Signal Processing and Integrated Networks (SPIN), pp.291-296, 20-21 Feb. 2014. 
[13]Jiang, R.; Zhu, Y.; He, T.; Liu, Y.; Ni, L.M., Exploiting Trajectory-Based Coverage for Geocast in Vehicular Networks. IEEE Transactions on Parallel and Distributed Systems, 2014, 25(12), 3177-3189.

[14]Imielinski T., Navas J. "GPS-Based Addressing and Routing," Internet Engineering Task Force (IETF), Network Working Group, Internet Draft, RFC 2009, 1996.

[15]Panichpapiboon, S., \& Pattara-Atikom, W. Connectivity requirements for self-organizing traffic information systems. Vehicular Technology, IEEE Transactions on, 57(6), 3333-3340, 2008.

[16]Li, G., Ma, M., Liu, C., \& Shu, Y. Adaptive fuzzy multiple attribute decision routing in VANETs. International Journal of Communication Systems. pp. 1-20, 2015.

[17]Daniels, R. C., \& Heath Jr, R. W. (2012). Link adaptation with position/motion information in vehicle-tovehicle networks. Wireless Communications, IEEE Transactions on, 11(2), 505-509.

[18] Yan, G., \& Olariu, S. (2011). A probabilistic analysis of link duration in vehicular ad hoc networks. Intelligent Transportation Systems, IEEE Transactions on, 12(4), 1227-1236.

[19]Sou, S. I., \& Tonguz, O. K. (2011). Enhancing VANET connectivity through roadside units on highways. Vehicular Technology, IEEE Transactions on, 60(8), 3586-3602.

[20] Yousefi, S., Altman, E., El-Azouzi, R., \& Fathy, M. (2008). Improving connectivity in vehicular ad hoc networks: An analytical study. Computer communications, 31(9), 1653-1659.

[21]Liu, P., Matolak, D. W., Ai, B., \& Sun, R. (2014). Path Loss Modeling for Vehicle-to-Vehicle Communication on a Slope. Vehicular Technology, IEEE Transactions on, 63(6), 2954-2958.

[22]Jin, W. L., \& Recker, W. W. (2010). An analytical model of multihop connectivity of inter-vehicle communication systems. Wireless Communications, IEEE Transactions on, 9(1), 106-112.

[23] Changle Li, Mengmeng Wang, and Lina Zhu, "Connectivity-Sensed Routing Protocol for Vehicular Ad Hoc Networks: Analysis and Design," International Journal of Distributed Sensor Networks, vol. 15, no.1, pp. 1$11,2015$.

[24] Ghafoor, H., Koo, I., \& Gohar, N. U. D. (2014). Neighboring and connectivity-aware routing in VANETs. The Scientific World Journal, 2014.

[25] Yang, Q., Lim, A., Li, S., Fang, J., \& Agrawal, P. ACAR: Adaptive connectivity aware routing for vehicular ad hoc networks in city scenarios.Mobile Networks and Applications, 15(1), 36-60, 2010.

[26] Alsharif, N., \& Shen, X. S. iCARII: Intersection-based connectivity aware routing in vehicular networks. In Communications (ICC), 2014 IEEE International Conference on (pp. 2731-2735). IEEE, 2014.

[27] Yousefi, S., Altman, E., El-Azouzi, R., \& Fathy, M. Analytical model for connectivity in vehicular ad hoc networks. Vehicular Technology, IEEE Transactions on, 57(6), 3341-3356, 2008.

[28] Shao, C., Leng, S., Zhang, Y., Vinel, A., \& Jonsson, M. Performance Analysis of Connectivity Probability and Connectivity-aware MAC Protocol Design for Platoon-based VANETs, 2015.

[29] Chandrasekharamenon, N. P., \& AnchareV, B. Connectivity analysis of one-dimensional vehicular ad hoc networks in fading channels. EURASIP Journal on Wireless Communications and Networking, 2012(1), 1-16, 2012.

[30] Alshaer, H. Securing vehicular ad-hoc networks connectivity with roadside units support. In GCC Conference and Exhibition (GCCCE), 2015 IEEE 8th (pp. 1-6). IEEE, 2015.

[31] Baskaran, R., Basha, M. S., Amudhavel, J., Kumar, K. P., Kumar, D. A., \& Vijayakumar, V.A bio-inspired artificial bee colony approach for dynamic independent connectivity patterns in VANET. In Circuit, Power and Computing Technologies (ICCPCT), 2015 International Conference on (pp. 1-6). IEEE, 2015.

[32]Rawat, D. B., \& Shetty, S. Enhancing connectivity for spectrum-agile vehicular ad hoc networks in fading channels. In Intelligent Vehicles Symposium Proceedings, 2014 IEEE (pp. 957-962). IEEE, 2014.

[33]Rawat, D. B., Bista, B. B., Yan, G., \& Olariu, S. Vehicle-to-vehicle connectivity and communication framework for vehicular ad-hoc networks. In Complex, Intelligent and Software Intensive Systems (CISIS), 2014 Eighth International Conference on (pp. 44-49). IEEE, 2014.

[34] Yan, T., Zhang, W. and Wang, G. "A grid-based on-road localization system in VANET with linear error propagation," IEEE Transactions on Wireless Communications, 13(2), 861-875, 2014.

[35] Shao, C., Leng, S., Zhang, Y., Vinel, A. and Jonsson, M. "Analysis of connectivity probability in platoonbased Vehicular Ad Hoc Networks," In Proceedings on Wireless Communications and Mobile Computing Conference (IWCMC), 2014 International, IEEE, pp. 706-711, 2014.

[36] O. Kaiwartya, S. Kumar, D. K. Lobiyal, A. H. Abdullah and A. N. Hassan, "Performance improvement in geographic routing for vehicular Ad Hoc networks," Sensors, 14(12), pp. 22342-22371, 2014. 


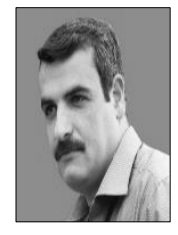

Ahmed Nazar Hassan is currently a Ph.D. candidate in Pervasive Computing Research Group Faculty of Computing Universiti Teknologi Malaysia (UTM), Skudai Johor, Malaysia. His research interest includes VANETs, WSNs and Computing.

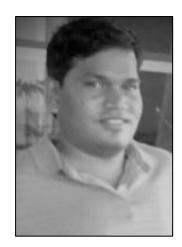

Omprakash Kaiwartya received his Ph.D., degree in Computer Science from School of Computer and Systems Sciences, Jawaharlal Nehru University, New Delhi, India in 2015. He is currently a Postdoctoral Research Fellow at Faculty of Computing, Universiti Teknologi Malaysia (UTM), Johor Bahru, Malaysia. His research interests focus on Vehicular Ad-hoc Networks, Mobile Ad-hoc Networks and Wireless Sensor Networks.

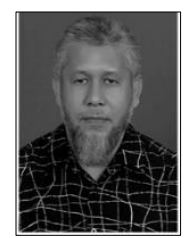

Abdul Hanan Abdullah received his Ph.D. degree from Aston University in Birmingham, United Kingdom in 1995. He is currently working as a Professor at Faculty of Computing, Universiti Teknologi Malaysia, Johor Bahru, Malaysia. He was the dean at the faculty from 2004 to 2011. Currently he is heading Pervasive Computing Research Group, a research group under K-Economy Research Alliances. His research interests include Wireless Sensor Networks, Vehicular Adhoc Networks, Internet of Vehicles, Network Security and Next Generation Networks.

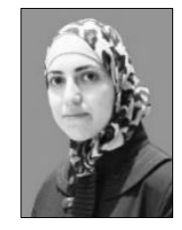

Dalya Khalid Sheet is currently a Ph.D. candidate in Pervasive Computing Research Group, Faculty of Computing, Universiti Teknologi Malaysia (UTM), Skudai Johor, Malaysia. Her research interests include Vehicular Ad-hoc Networks and Wireless Sensor Networks.

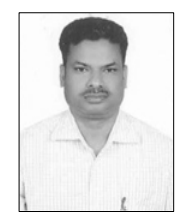

Ram Shringar Raw received his Ph.D degree in Computer Science from School of Computer and Systems Sciences, Jawaharlal Nehru University, New Delhi, India in 2011. He is currently working as Associate Professor at Department of Computer Science, IGNTU, Central University, India. His research interest includes Mobile Ad hoc Networks, Vehicular Ad hoc Networks and Wireless Sensor Networks. 